\title{
EXTENSION ALGEBRAS OF CUNTZ ALGEBRA, II
}

\author{
SHUDONG LIU ${ }^{\otimes}$ and XIAOCHUN FANG
}

\author{
(Received 11 August 2008)
}

\begin{abstract}
In this paper, we construct the unique (up to isomorphism) extension algebra, denoted by $E_{\infty}$, of the Cuntz algebra $\mathcal{O}_{\infty}$ by the $C^{*}$-algebra of compact operators on a separable infinite-dimensional Hilbert space. We prove that two unital monomorphisms from $E_{\infty}$ to a unital purely infinite simple $C^{*}$-algebra are approximately unitarily equivalent if and only if they induce the same homomorphisms in K-theory.
\end{abstract}

2000 Mathematics subject classification: primary 46L05.

Keywords and phrases: $C^{*}$-algebra, extension, Cuntz algebra.

\section{Introduction and preliminaries}

The study of extension theory for $C^{*}$-algebras was initiated by Busby [5] and further developed by Brown et al. [3, 4]. It provides an important way to construct new $C^{*}$-algebras.

Let $A$ be a separable $C^{*}$-algebra. An extension of $A$ by $\mathcal{K}$, the $C^{*}$-algebra of compact operators on a separable infinite-dimensional Hilbert space $H$, is a short exact sequence

$$
0 \longrightarrow \mathcal{K} \stackrel{i}{\longrightarrow} E \stackrel{q}{\longrightarrow} A \longrightarrow 0
$$

of $C^{*}$-algebras. The Busby invariant $\tau: A \rightarrow \mathcal{Q}(H)=B(H) / \mathcal{K}$ of the above extension is the unique homomorphism for which there is a commutative diagram

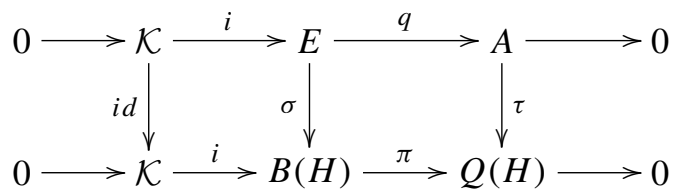

We also refer to $\tau$ as an extension of $A$ by $\mathcal{K}$ and call $E$ an extension algebra of $A$ (see [1,9] for details). The invariant $\tau$ is said to be essential if $\tau$ is injective, and

This work was supported by the National Natural Science Foundation of China (grant no. 10771161) and the Natural Science Foundation of Shandong Province (grant no. Y2006A03).

(C) 2009 Australian Mathematical Society 0004-9727/2009 \$16.00 
trivial if $\tau$ lifts to a homomorphism from $A$ to $B(H)$, which will be the case if and only if the associated exact sequence splits.

If $A$ is unital, an extension $\tau: A \rightarrow \mathcal{Q}(H)$ is called unital if $\tau$ is unital. A trivial extension $\tau$ is called strongly unital if $\tau$ lifts to a unital homomorphism from $A$ to $B(H)$.

Let $\tau_{1}, \tau_{2}: A \rightarrow \mathcal{Q}(H)$ be two extensions of $A$. Then $\tau_{1}$ and $\tau_{2}$ are said to be strongly equivalent if there is a unitary $u \in B(H)$ such that $\tau_{2}(a)=\pi(u) \tau_{1}(a) \pi(u)^{*}$ for all $a \in A$. Let $\mathbf{E x t}_{\mathrm{s}}(A)$ be the set of strong equivalence classes of essential extensions of $A$. If $A$ is unital, we denote by $\mathbf{E x t}_{\mathrm{s}}^{\mathrm{u}}(A)$ the set of strong equivalence classes of unital essential extensions of $A$. We define the sum of $\tau_{1}$ and $\tau_{2}$ by $\tau_{1} \oplus \tau_{2}: A \rightarrow \mathcal{Q}(H) \oplus \mathcal{Q}(H) \subset M_{2}(\mathcal{Q}(H)) \cong \mathcal{Q}(H)$. Then $\mathbf{E x t}_{\mathrm{s}}(A)$ and $\mathbf{E x t}_{\mathrm{s}}^{\mathrm{u}}(A)$ are semigroups. Let $\operatorname{Ext}(A)$ be the quotient of $\operatorname{Ext}_{\mathrm{s}}(A)$ by the subsemigroup of essential trivial extensions.

Extensions of Cuntz algebras were first studied by Cuntz [6, 7], and further investigations were undertaken by Paschke and Salinas [15], Lin [8, 11], and the authors [12]. In [12], we constructed and classified all extension algebras of the Cuntz algebra $\mathcal{O}_{n}$ for $n \geq 2$. In this paper, we consider the case of $\mathcal{O}_{\infty}$, and show that there is only one extension algebra (up to isomorphism) of $\mathcal{O}_{\infty}$, which we denote by $E_{\infty}$. We construct this extension algebra and describe its K-theory. We also prove that two unital monomorphisms from $E_{\infty}$ to a unital purely infinite simple $C^{*}$-algebra are approximately unitarily equivalent if and only if they induce the same homomorphisms in K-theory.

Throughout this paper, $\mathbb{Z}$ will denote the group of integers and $\mathbb{Z}_{+}$the semigroup of nonnegative integers; we write $\mathbb{Z}_{n}=\mathbb{Z} / n \mathbb{Z}$ and $\mathbb{Z}^{n}=\mathbb{Z} \oplus \cdots \oplus \mathbb{Z}(n$ copies of $\mathbb{Z})$.

\section{Extension algebras of $\mathcal{O}_{\infty}$}

Recall that the Cuntz algebra $\mathcal{O}_{\infty}$ is the universal $C^{*}$-algebra generated by a sequence of isometries $\left\{s_{i}\right\}$ with mutually orthogonal range projections. It is wellknown that $\mathcal{O}_{\infty}$ is a unital purely infinite simple $C^{*}$-algebra and that $K_{0}\left(\mathcal{O}_{\infty}\right)=\mathbb{Z}$ and $K_{1}\left(\mathcal{O}_{\infty}\right)=0$. Also, $\mathcal{O}_{\infty}$ is unique in the sense that for any sequence of isometries $\left\{s_{i}^{\prime}\right\}$ with mutually orthogonal range projections, the $C^{*}$-algebra generated by $\left\{s_{i}^{\prime}\right\}$ is isomorphic to $\mathcal{O}_{\infty}$.

A separable nuclear $C^{*}$-algebra $A$ is said to satisfy the $U C T[1,9]$ if for any separable $C^{*}$-algebra $B$ there is the short exact sequence

$$
0 \longrightarrow \operatorname{Ext}_{\mathbb{Z}}^{1}\left(K_{*}(A), K_{*}(B)\right) \stackrel{\delta}{\longrightarrow} K K^{*}(A, B) \stackrel{\gamma}{\longrightarrow} \operatorname{Hom}\left(K_{*}(A), K_{*}(B)\right) \longrightarrow 0 .
$$

Here the map $\gamma$ has degree zero and $\delta$ has degree one. In particular, taking $B=\mathcal{K}$, since $\operatorname{Ext}(A, B)=K K(A, S B)$ we have the following short exact sequence:

$$
0 \longrightarrow \operatorname{Ext}_{\mathbb{Z}}^{1}\left(K_{0}(A), \mathbb{Z}\right) \longrightarrow \operatorname{Ext}(A) \longrightarrow \operatorname{Hom}\left(K_{1}(A), \mathbb{Z}\right) \longrightarrow 0 .
$$


Proposition 2.1. $\mathbf{E x t}_{\mathrm{s}}^{\mathrm{u}}\left(\mathcal{O}_{\infty}\right)=0$.

Proof. Since $\mathcal{O}_{\infty}$ is a unital separable nuclear $C^{*}$-algebra, $\operatorname{Ext}\left(\mathcal{O}_{\infty}\right)$ is a group. Since $K_{0}\left(\mathcal{O}_{\infty}\right)=\mathbb{Z}$ and $K_{1}\left(\mathcal{O}_{\infty}\right)=0, \operatorname{Ext}\left(\mathcal{O}_{\infty}\right)=0$ by the $U C T$. Because $\left[\mathbf{1}_{\mathcal{O}_{\infty}}\right]_{0}$ is a generator of $K_{0}\left(\mathcal{O}_{\infty}\right)=\mathbb{Z}$,

$$
\left\{h\left(\left[\mathbf{1}_{\mathcal{O}_{\infty}}\right]_{0}\right) \mid h \in \operatorname{Hom}\left(K_{0}\left(\mathcal{O}_{\infty}\right), \mathbb{Z}\right)\right\}=\mathbb{Z} .
$$

Since $\mathcal{O}_{\infty} \otimes C(\mathbb{T})$ satisfies the $U C T$, it follows from [2, Proposition 1] that

$$
\operatorname{Ext}_{\mathrm{s}}^{\mathrm{u}}\left(\mathcal{O}_{\infty}\right)=\mathbb{Z} /\left\{h\left(\left[\mathbf{1}_{\mathcal{O}_{\infty}}\right]_{0}\right) \mid h \in \operatorname{Hom}\left(K_{0}\left(\mathcal{O}_{\infty}\right), \mathbb{Z}\right)\right\}=0 .
$$

The following lemma is well-known, but we give its proof for completeness.

Lemma 2.2. Let $A$ be a (unital) separable $C^{*}$-algebra, and let

$$
0 \longrightarrow \mathcal{K} \stackrel{i_{1}}{\longrightarrow} E_{1} \stackrel{q_{1}}{\longrightarrow} A \longrightarrow 0
$$

and

$$
0 \longrightarrow \mathcal{K} \stackrel{i_{2}}{\longrightarrow} E_{2} \stackrel{q_{2}}{\longrightarrow} A \longrightarrow 0
$$

be two (unital) essential extensions of $A$ with Busby invariants $\tau_{1}$ and $\tau_{2}$, respectively. Then $\tau_{1}$ and $\tau_{2}$ are strongly equivalent if and only if there is an isomorphism $\varphi: E_{1} \rightarrow$ $E_{2}$ such that the following diagram is commutative:

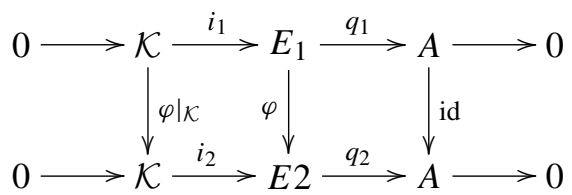

PROOF. If $\tau_{1}$ and $\tau_{2}$ are strongly equivalent, then there is a unitary $u \in B(H)$ such that

$$
\tau_{2}(x)=\pi(u) \tau_{1}(x) \pi(u)^{*} \quad \forall x \in A .
$$

Define $\varphi: E_{1} \rightarrow E_{2}$ by $\varphi(x)=u x u^{*}$. Now, for $x \in E_{1}$,

$$
\begin{aligned}
\pi(\varphi(x)) & =\pi\left(u x u^{*}\right)=\pi(u) \pi(x) \pi(u)^{*} \\
& =\pi(u) \tau_{1}\left(q_{1}(x)\right) \pi(u)^{*}=\tau_{2}\left(q_{1}(x)\right),
\end{aligned}
$$

so $\varphi(x) \in E_{2}$. Therefore $\varphi$ is well-defined and it is obvious that $\varphi$ is an isomorphism. Since $\tau_{2}\left(q_{2}(\varphi(x))\right)=\pi(\varphi(x))=\tau_{2}\left(q_{1}(x)\right)$ and $\tau_{2}$ is injective, we have $q_{2}(\varphi(x))=$ $q_{1}(x)$. Thus, the above diagram is commutative.

Conversely, suppose that there is an isomorphism $\varphi: E_{1} \rightarrow E_{2}$ such that the diagram is commutative. It is well-known that $\left.\varphi\right|_{\mathcal{K}}$ is an isomorphism from $\mathcal{K}$ onto $\mathcal{K}$ and there is a unitary $u \in B(H)$ such that $\varphi(x)=u x u^{*}$ for all $x \in \mathcal{K}$. For any $x \in \mathcal{K}$ and $y \in E_{1}$,

$$
\varphi(y)\left(u x u^{*}\right)=\varphi(y x)=u y x u^{*}=\left(u y u^{*}\right)\left(u x u^{*}\right) .
$$


Since the range of $u \mathcal{K} u^{*}$ is dense in $H, \varphi(y)=u y u^{*}$. For $x=q_{1}(y) \in A$ with $y \in E_{1}$,

$$
\begin{aligned}
\pi(u) \tau_{1}(x) \pi(u)^{*} & =\pi(u) \tau_{1}\left(q_{1}(y)\right) \pi(u)^{*} \\
& =\pi(u) \pi(y) \pi(u)^{*}=\pi(\varphi(y)) \\
& =\tau_{2}\left(q_{2}(\varphi(y))\right)=\tau_{2}\left(q_{1}(y)\right)=\tau_{2}(x) .
\end{aligned}
$$

By Proposition 2.1 and Lemma 2.2, there exists a unique (up to isomorphism) extension algebra of $\mathcal{O}_{\infty}$. We now construct it concretely.

Let $\left\{t_{i} \mid i=1,2, \ldots\right\}$ be a sequence of partial isometries with mutually orthogonal range projections such that $t_{1}^{*} t_{1}<1, t_{i}^{*} t_{i}=1$ for $i \geq 2$, and $p+\sum_{i=1}^{n} t_{i} t_{i}^{*}<1$ for any positive integer $n$, where $p=1-t_{1}^{*} t_{1}$ is a projection of rank one. Let $E_{\infty}$ be the universal $C^{*}$-algebra generated by $\left\{t_{i} \mid i=1,2, \ldots\right\}$, and let $I\left(E_{\infty}\right)$ be the (only closed) ideal of $E_{\infty}$ generated by $p$.

TheOREM 2.3. $I\left(E_{\infty}\right) \cong \mathcal{K}$ and $E_{\infty} / I\left(E_{\infty}\right) \cong \mathcal{O}_{\infty}$; that is, $E_{\infty}$ is an essential unital extension of $\mathcal{O}_{\infty}$ by $\mathcal{K}$.

PROOF. Let $l$ be a positive integer and $W_{l}$ the set of all $l$-tuples $\left(i_{1}, \ldots, i_{l}\right)$ with $i_{j} \in \mathbb{N}=\{1,2, \ldots\},, j=1, \ldots, l$. We assume that $W_{0}=\{0\}$ and $W=\bigcup_{l \geq 0} W_{l}$. Let $T_{0}=1$ and, for $\alpha=\left(i_{1}, \ldots, i_{l}\right) \in W_{l}$, denote by $T_{\alpha}$ the partial isometry $T_{\alpha}=$ $t_{i_{1}} \cdots t_{i_{l}}$. Note that $p T_{\alpha}=0$ for any $\alpha \in W$.

Let $\mathcal{F}$ be the set of all linear combinations of elements of the form $T_{\alpha} p T_{\beta}^{*}$ for any $\alpha, \beta \in W$. It is easy to see that the closure of $\mathcal{F}$ is an ideal of $E_{\infty}$, and that $\mathcal{F}$ is contained in every ideal containing $p$. It follows that the closure of $\mathcal{F}$ is the ideal of $E_{\infty}$ generated by $p$, that is, $I\left(E_{\infty}\right)$.

Let $X=\left(T_{\alpha} p T_{\mu}^{*}\right)\left(T_{\nu} p T_{\beta}^{*}\right)$. It is easy to see that $X \neq 0$ if and only if $\mu=v$. One can check that

$$
\left(T_{\alpha} p T_{\mu}^{*}\right)\left(T_{\nu} p T_{\beta}^{*}\right)=\delta_{\mu \nu} T_{\alpha} p T_{\beta}^{*}
$$

and

$$
\left(T_{\alpha} p T_{\beta}^{*}\right)^{*}=T_{\beta} p T_{\alpha}^{*} .
$$

Then the set $\left\{T_{\alpha} p T_{\beta}^{*} \mid \alpha, \beta \in W\right\}$ is a self-adjoint system of matrix units generating $\mathcal{F}$. It follows that $I\left(E_{\infty}\right)$ is isometric to $\mathcal{K}$.

Let $\pi: E_{\infty} \rightarrow E_{\infty} / I\left(E_{\infty}\right)$ be the quotient map. It is easy to see that $\left\{\pi\left(t_{i}\right)\right\}$ is a sequence of isometries in $E_{\infty} / I\left(E_{\infty}\right)$ with orthogonal ranges. Since $E_{\infty} / I\left(E_{\infty}\right)$ is a unital $C^{*}$-algebra generated by $\left\{\pi\left(t_{i}\right)\right\}, E_{\infty} / I\left(E_{\infty}\right) \cong \mathcal{O}_{\infty}$ by the uniqueness of $\mathcal{O}_{\infty}$. Therefore $E_{\infty}$ is an essential unital extension of $\mathcal{O}_{\infty}$ by $\mathcal{K}$, since $\mathcal{O}_{\infty}$ is a unital simple $C^{*}$-algebra.

REMARK 2.4. Let $H$ be a separable infinite-dimensional Hilbert space. It is easy to see that there exist partial isometries $t_{1}, t_{2}, \ldots$ in $B(H)$ satisfying the conditions in the definition of $E_{\infty}$.

Corollary 2.5 (Uniqueness of $E_{\infty}$ ). Let $\left\{t_{i}^{\prime} \mid i=1,2, \ldots\right\}$ be a sequence of partial isometries with mutually orthogonal range projections such that $t_{1}^{\prime *} t_{1}^{\prime}<1$, 
$t^{\prime *} t_{i}^{\prime}=1$ for $i \geq 2$, and $p^{\prime}+\sum_{i=1}^{n} t_{i}^{\prime} t_{i}^{\prime *}<1$ for any positive integer $n$, where $p^{\prime}=$ $1-t_{1}^{\prime *} t_{1}^{\prime}$ is a projection of rank one. Then the $C^{*}$-algebra $E$ generated by $\left\{t_{i}^{\prime} \mid i=\right.$ $1,2, \ldots\}$ is isomorphic to $E_{\infty}$. In other words, $E_{\infty}$ is independent of the choice of the generators.

PRoOF. As in Theorem 2.3, we may prove that the ideal $I$ of $E$ generated by the projection $p^{\prime}$ is isomorphic to $\mathcal{K}$ and that $E / I \cong \mathcal{O}_{\infty}$. Since there is only one extension algebra of $\mathcal{O}_{\infty}$, we have $E \cong E_{\infty}$.

THEOREM 2.6. $K_{0}\left(E_{\infty}\right)=\mathbb{Z} \oplus \mathbb{Z}$ and $K_{1}\left(E_{\infty}\right)=0$.

PROOF. By Theorem 2.3, we have the short exact sequence

$$
0 \longrightarrow \mathcal{K} \longrightarrow E_{\infty} \longrightarrow \mathcal{O}_{\infty} \longrightarrow 0
$$

By the six-term exact sequence of K-theory, we have the following commutative diagram:

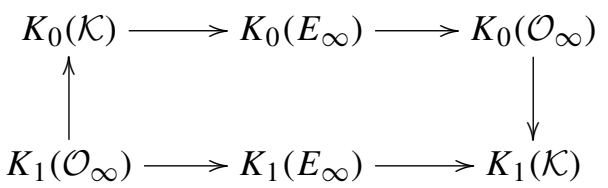

Since $K_{0}\left(\mathcal{O}_{\infty}\right)=\mathbb{Z}$ and $K_{1}\left(\mathcal{O}_{\infty}\right)=0, K_{1}\left(E_{\infty}\right)=0$ and the sequence

$$
0 \longrightarrow \mathbb{Z} \longrightarrow K_{0}\left(E_{\infty}\right) \longrightarrow \mathbb{Z} \longrightarrow 0
$$

is exact. Therefore $K_{0}\left(E_{\infty}\right) \cong \mathbb{Z} \oplus \mathbb{Z}$.

Let $A$ be a unital $C^{*}$-algebra. Two projections $p$ and $q$ in $A$ are said to be (Murray-von Neumann) equivalent, denoted by $p \sim q$, if there is a partial isometry $v \in A$ such that $v^{*} v=p$ and $v v^{*}=q$; we let $[p]$ denote the equivalence class of projections in $A$ containing $p$. The projections $p$ and $q$ are called unitarily equivalent, denoted by $p \sim_{\mathrm{u}} q$, if there is a unitary $u$ in $A$ such that $u^{*} p u=q ;[p]_{\mathrm{u}}$ denotes the unitary equivalence class of projections in $A$ containing $p$. Moreover, $p$ and $q$ are called homotopically equivalent, denoted by $p \sim_{\mathrm{h}} q$, if $p$ and $q$ are in the same path component of projections in $A ;[p]_{\mathrm{h}}$ denotes the homotopic equivalence class of projections in $A$ containing $p$.

The following proposition can be obtained immediately from results in [13] and [14].

PROPOSITION 2.7.

(1) $K_{0}\left(E_{\infty}\right)=\left\{[p] \mid p \in E_{\infty} \backslash \mathcal{K}\right.$ is a projection $\}$

$=\left\{[p] \mid p \in E_{\infty} \backslash \mathcal{K}\right.$ is a projection and $\left.1-p \in E_{\infty} \backslash \mathcal{K}\right\}$

$=\left\{[p]_{\mathrm{h}} \mid p \in E_{\infty} \backslash \mathcal{K}\right.$ is a projection and $\left.1-p \in E_{\infty} \backslash \mathcal{K}\right\}$

$=\left\{[p]_{\mathrm{u}} \mid p \in E_{\infty} \backslash \mathcal{K}\right.$ is a projection and $\left.1-p \in E_{\infty} \backslash \mathcal{K}\right\}$.

(2) Let $p$ and $q$ be projections in $E_{\infty} \backslash \mathcal{K}$ such that $1-p$ and $1-q \in E_{\infty} \backslash \mathcal{K}$. Then $p \sim q, p \sim_{\mathrm{u}} q$ and $p \sim_{\mathrm{h}} q$ are equivalent. 
Recall that $E_{n}$ is the universal $C^{*}$-algebra generated by isometries $t_{1}, t_{2}, \ldots, t_{n}$ with $\sum_{i=1}^{n} t_{i} t_{i}^{*}<1$. It is well-known that $E_{n}$ is a unital essential extension of $\mathcal{O}_{n}$ by $\mathcal{K}$ and that $\mathcal{O}_{\infty}=\lim _{\rightarrow} E_{n}$.

Let $F_{n}(n \geq 2)$ be the $C^{*}$-subalgebra of $E_{\infty}$ generated by $t_{1}, t_{2}, \ldots, t_{n}$, and let $I\left(F_{n}\right)$ be the ideal of $F_{n}$ generated by $p=1-t_{1}^{*} t_{1}$. We can prove the following results as above, so the details are omitted.

PROPOSITION 2.8.

(1) $\operatorname{Ext}_{\mathrm{s}}^{\mathrm{u}}\left(E_{n}\right)=0$.

(2) $I\left(F_{n}\right) \cong \mathcal{K}$ and $F_{n} / I\left(F_{n}\right) \cong E_{n}$; moreover, $F_{n}$ is the only unital essential extension algebra of $E_{n}$ by $\mathcal{K}$.

(3) $K_{0}\left(F_{n}\right)=\mathbb{Z} \oplus \mathbb{Z}$ and $K_{1}\left(F_{n}\right)=0$.

(4) $E_{\infty}=\lim _{\longrightarrow} F_{n}$, where the map $F_{n} \rightarrow F_{n+1}$ of the system sends $t_{i}$ to $t_{i}$ for $i=$ $1,2, \ldots, n$.

Corollary 2.9. Let $H$ be a separable infinite-dimensional Hilbert space. Suppose that $\varphi, \psi: A \rightarrow Q(H)$ are two unital injective homomorphisms, where $A=\mathcal{O}_{\infty}$ or $E_{n}$. Then there is a unitary $u \in B(H)$ such that $\varphi(x)=\pi(u)^{*} \psi(x) \pi(u)$ for all $x \in A$.

Proof. Now, $\varphi$ and $\psi$ are two unital essential extensions of $A$ by $\mathcal{K}$, so the result follows immediately from the fact that $\operatorname{Ext}_{s}^{\mathrm{u}}(A)=0$.

Corollary 2.10. Let $H$ be a separable infinite-dimensional Hilbert space.

(1) Let $v_{1}, v_{2}, \ldots, v_{n}(n \geq 2)$ be isometries in the Calkin algebra $Q(H)$ with mutually orthogonal range projections such that $\sum_{i=1}^{n} t_{i} t_{i}^{*}<1$. Then there are isometries $V_{1}, V_{2}, \ldots, V_{n}$ in $B(H)$ with mutually orthogonal range projections such that $\pi\left(V_{i}\right)=v_{i}$ for $i=1,2, \ldots, n$.

(2) Let $\left\{v_{i}\right\}$ be a sequence of isometries in $Q(H)$ with mutually orthogonal range projections. Then there is a sequence of isometries $\left\{V_{i}\right\}$ in $B(H)$ with mutually orthogonal range projections such that $\pi\left(V_{i}\right)=v_{i}$ for $i=1,2, \ldots$

Proof. For part (1), the map $\tau: E_{n} \rightarrow Q(H)$ defined by $\tau\left(t_{i}\right)=v_{i}$ is a unital essential extension of $E_{n}$ by $\mathcal{K}$. It is trivial since $\operatorname{Ext}_{\mathrm{s}}^{\mathrm{u}}\left(E_{n}\right)=0$; thus $\tau$ lifts to a unital injective homomorphism $\sigma: E_{n} \rightarrow B(H)$. Now put $V_{i}=\sigma\left(t_{i}\right)$. The proof of (2) is similar, since $\operatorname{Ext}_{\mathrm{s}}^{\mathrm{u}}\left(\mathcal{O}_{\infty}\right)=0$.

\section{Homomorphisms from $E_{\infty}$}

Definition 3.1. Let $A$ and $B$ be $C^{*}$-algebras, let $F$ be a finite subset of $A$, and let $\varphi$ and $\psi$ be two homomorphisms from $A$ into $B$. Let $\varepsilon>0$. We say that $\varphi$ and $\psi$ are approximately unitarily equivalent within $\varepsilon$, with respect to $F$, if there is a unitary $u \in \tilde{B}$ such that

$$
\left\|\varphi(x)-u \psi(x) u^{*}\right\|<\varepsilon
$$


for all $x \in F$, where $\tilde{B}$ is the unitization of $B$. We abbreviate this as $\varphi \sim_{\varepsilon} \psi$ with respect to $F$. When the set $F$ is understood, we shall omit mention of it.

We further say that $\varphi$ and $\psi$ are approximately unitarily equivalent if for every finite $F \subset A$ and $\varepsilon>0$, we have $\varphi \sim_{\varepsilon} \psi$ with respect to $F$.

THEOREM 3.2. Let $A$ be a unital purely infinite simple $C^{*}$-algebra, and let $\varphi, \psi$ : $E_{\infty} \rightarrow A$ be two unital monomorphisms. Then the following statements are equivalent:

(i) $\varphi$ and $\psi$ are approximately unitarily equivalent;

(ii) $[\varphi]=[\psi]$ in $K K\left(E_{\infty}, A\right)$;

(iii) $\varphi_{*}=\psi_{*}: K_{0}\left(E_{\infty}\right) \rightarrow K_{0}(A)$;

(iv) $\varphi(p) \sim \psi(p)$ in $A$, where $p=1-t_{1}^{*} t_{1}$;

(v) $\varphi\left(t_{1}^{*} t_{1}\right) \sim \psi\left(t_{1}^{*} t_{1}\right)$ in $A$.

ProOF. (i) $\Leftrightarrow$ (ii). Since $K_{*}\left(E_{\infty}\right)$ is finitely generated, $K K\left(E_{\infty}, A\right)=K L\left(E_{\infty}, A\right)$. The result then follows immediately from [10, Theorem 2.9], since every unital purely infinite simple $C^{*}$-algebra has properties $\left(P_{1}\right),\left(P_{2}\right)$ and $\left(P_{3}\right)$ and every monomorphism into a simple $C^{*}$-algebra is full in the sense of [10].

(ii) $\Leftrightarrow$ (iii). Since $K_{0}\left(E_{\infty}\right)=\mathbb{Z} \oplus \mathbb{Z}$ is a free abelian group and $K_{1}\left(E_{\infty}\right)=0$, by the $U C T$ we have $K K\left(E_{\infty}, A\right) \cong \operatorname{Hom}\left(K_{0}\left(E_{\infty}\right), K_{0}(A)\right)$. Note that $\gamma(\varphi)=\varphi_{*}$. Therefore, $[\varphi]=[\psi]$ in $K K\left(E_{\infty}, A\right)$ if and only if $\varphi_{*}=\psi_{*}: K_{0}\left(E_{\infty}\right) \rightarrow K_{0}(A)$.

(iii) $\Leftrightarrow$ (iv). Since $K_{0}\left(E_{\infty}\right)=\mathbb{Z} \oplus \mathbb{Z}$ is an abelian group generated by [1] $]_{0}$ and $[p]_{0}$, $\varphi_{*}=\psi_{*}: K_{0}\left(E_{\infty}\right) \rightarrow K_{0}(A)$ if and only if $[\varphi(1)]_{0}=[\psi(1)]_{0}$ and $[\varphi(p)]_{0}=[\psi(p)]_{0}$ in $K_{0}(A)$; this occurs if and only if $[\varphi(p)]_{0}=[\psi(p)]_{0}$, since $\varphi$ and $\psi$ are unital. But $A$ is a purely infinite simple $C^{*}$-algebra, so $[\varphi(p)]_{0}=[\psi(p)]_{0}$ in $K_{0}(A)$ if and only if $\varphi(p) \sim \psi(p)$ in $A$.

(iv) $\Leftrightarrow(\mathrm{v})$. This is obvious since $\varphi(p), \psi(p), \varphi\left(t_{1}^{*} t_{1}\right)=1-\varphi(p)$ and $\psi\left(t_{1}^{*} t_{1}\right)=$ $1-\psi(p)$ are nonzero projections in a purely infinite simple $C^{*}$-algebra.

Similar results hold for $F_{n}$, with the same proof.

Proposition 3.3. Let $A$ be a unital purely infinite simple $C^{*}$-algebra, and let $\varphi, \psi: F_{n} \rightarrow A$ be two unital monomorphisms. Then the following statements are equivalent:

(i) $\varphi$ and $\psi$ are approximately unitarily equivalent;

(ii) $[\varphi]=[\psi]$ in $K K\left(F_{n}, A\right)$;

(iii) $\varphi_{*}=\psi_{*}: K_{0}\left(F_{n}\right) \rightarrow K_{0}(A)$;

(iv) $\varphi(p) \sim \psi(p)$ in $A$, where $p=1-t_{1}^{*} t_{1}$;

(v) $\varphi\left(t_{1}^{*} t_{1}\right) \sim \psi\left(t_{1}^{*} t_{1}\right)$ in $A$.

\section{References}

[1] B. Blackadar, K-Theory for Operator Algebras (Springer, New York, 1986).

[2] L. G. Brown and M. Dadarlat, 'Extensions of $C^{*}$-algebras and quasidiagonality', J. London Math. Soc. (2) 53 (1996), 582-600. 
[3] L. G. Brown, R. G. Douglas and P. A. Fillmore, 'Unitary equivalence modulo the compact operators and extensions of $C^{*}$-algebras', in: Proc. Conf. Operator Theory (Dalhousie Univ., Halifax, 1973), Lecture Notes in Mathematics, 345 (Springer, Berlin, 1973), pp. 58-128.

[4] - 'Extensions of $C^{*}$-algebras and K-homology', Ann. of Math. (2) 105(2) (1977), 265-324.

[5] R. C. Busby, 'Double centralizers and extensions of $C^{*}$-algebras', Trans. Amer. Math. Soc. 132 (1968), 79-99.

[6] J. Cuntz, 'Simple $C^{*}$-algebras generated by isometries', Comm. Math. Phys. 52(2) (1977), 173-185.

[7] - 'K-theory for certain $C^{*}$-algebras', Ann. of Math. (2) 113 (1981), 181-197.

[8] H. Lin, 'On the classification of $C^{*}$-algebras of real rank zero with zero $K_{1}$ ', J. Operator Theory 35 (1996), 147-178.

[9] - An Introduction to the Classification of Amenable $C^{*}$-algebras (World Scientific, Singapore, 2001).

[10] $\longrightarrow$,Full extension and approximate unitary equivalences', Pacific J. Math. 229(2) (2007), 389-428.

[11] H. Lin and N. C. Phillips, 'Approximate unitary equivalence of homomorphisms from $\mathcal{O}_{\infty}$ ', $J$. Reine Angew. Math. 464 (1995), 173-186.

[12] S. Liu and X. Fang, 'Extension algebras of Cuntz algebra', J. Math. Anal. Appl. 329 (2007), 655-663.

[13] $\quad$ K-theory for extensions of purely infinite simple $C^{*}$-algebras', Chinese Ann. of Math. Ser. A 29(2) (2008), 195-202.

[14] , 'K-theory for extensions of purely infinite simple $C^{*}$-algebras: II', Chinese Ann. of Math. Ser. A to appear.

[15] W. L. Paschke and N. Salinas, 'Matrix algebras over $\mathcal{O}_{n}$ ', Michigan Math. J. 26(1) (1979), 3-12.

SHUDONG LIU, School of Mathematical Sciences, Qufu Normal University, Qufu, People's Republic of China

e-mail: 1shd008@163.com

XIAOCHUN FANG, Department of Mathematics, Tongji University, Shanghai, People's Republic of China

e-mail: xfang@mail.tongji.edu.cn 